(C) 2021, The Authors. Published by Elsevier Inc. and Fass Inc. on behalf of the American Dairy Science Association ${ }^{\circledR}$. This is an open access article under the CC BY-NC-ND license (http://creativecommons.org/licenses/by-nc-nd/4.0/).

\title{
Identification and characterization of circular RNAs in mammary gland tissue from sheep at peak lactation and during the nonlactating period
}

\author{
Jiqing Wang, ${ }^{1 *}$ ๑ Huitong Zhou, ${ }^{1,2}$ Jon G. H. Hickford, ${ }^{1,2}$ Zhiyun Hao, ${ }^{1} \odot$ Hua Gong,,${ }^{1,2}$ Jiang Hu, ${ }^{1}$ Xiu Liu, ${ }^{1}$ \\ Shaobin Li, ${ }^{1}$ Jiyuan Shen, ${ }^{1} \mathrm{Na} \mathrm{Ke},{ }^{1}$ Yize Song, ${ }^{1}$ Lirong Qiao, ${ }^{1}$ and Yuzhu Luo ${ }^{1 *}$ \\ ${ }^{1}$ Gansu Key Laboratory of Herbivorous Animal Biotechnology, Faculty of Animal Science and Technology, Gansu Agricultural University, \\ Lanzhou 730070, China \\ ${ }^{2}$ Gene-Marker Laboratory, Faculty of Agriculture and Life Sciences, Lincoln University, Lincoln 7647, New Zealand
}

\section{ABSTRACT}

Circular RNAs are a class of noncoding RNA with a widespread occurrence in eukaryote tissues, and with some having been demonstrated to have clear biological function. In sheep, little is known about the role of circular RNAs in mammary gland tissue, and therefore an RNA sequencing approach was used to compare mammary gland tissue expression of circular RNAs in 9 Small Tail Han sheep at peak lactation, and subsequently when they were not lactating. These 9 sheep had their RNA pooled for analysis into 3 libraries from peak lactation and 3 from the nonlactating period. A total of 3,278 and 1,756 circular RNAs were identified in the peak lactation and nonlactating mammary gland tissues, respectively, and the expression and identity of 9 of them was confirmed using reverse transcriptasepolymerase chain reaction analysis and DNA sequencing. The type, chromosomal location and length of the circular RNAs identified were ascertained. Forty upregulated and one downregulated circular RNAs were characterized in the mammary gland tissue at peak lactation compared with the nonlactating mammary gland tissue. Gene ontology enrichment analysis revealed that the parental genes of these differentially expressed circular RNAs were related to molecular function, binding, protein binding, ATP binding, and ion binding. Five differentially expression circular RNAs were selected for further analysis to predict their target microRNAs, and some microRNAs reportedly associated with the development of the mammary gland were found in the constructed circular RNA-microRNA network. This study reveals the expression profiles and characterization of circular RNAs at 2 key stages of mammary gland

Received May 16, 2020.

Accepted September 7, 2020.

*Corresponding authors: wangjq@gsau.edu.cn and luoyz@gsau.edu .cn activity, thereby providing an improved understanding of the roles of circular RNAs in the mammary gland of sheep.

Key words: circular RNA, mammary gland, sheep, RNA sequencing

\section{INTRODUCTION}

Circular RNAs are a kind of noncoding RNA. Although the first circular RNA molecules were discovered in plant viroids as early as 1976 (Sanger et al., 1976), they were assumed to be the product of aberrant RNAsplicing or molecular flukes; thus, they were ignored for a long time. It is now more widely accepted that circular RNAs can be produced by protein-coding genes and that their occurrence is widespread in eukaryotic cells (Wilusz, 2018). These circular RNAs are found to be generated from back-splicing reactions, in which a downstream $5^{\prime}$ splice site (splice-donor) is joined to an upstream $3^{\prime}$ splice site (splice-acceptor), or by the direct linkage of the $5^{\prime}$ and $3^{\prime}$ ends of linear RNAs (Lasda and Parker, 2014). They are stable entities and they can accumulate in the cytoplasm. The great majority of circular RNAs are expressed at low levels, but some have 10-fold higher levels of expression than their recognized linear mRNAs (Jeck et al., 2013).

Although the function of the vast majority of circular RNAs remains unclear, some circular RNAs have molecular functions that affect phenotype. For example, some can function as microRNA sponges, and it has been described how they can result in a reduction in microRNA activity, with an accompanying increase in the level of expression of the microRNA targets in mouse brain (Hansen et al., 2013) and in human HEK293 cells (Memczak et al., 2013). Additionally, the knockdown of some circular RNAs can result in decreased expression of their parent genes (Zhang et al., 2013), and this suggests a cis-regulatory role in gene expression. In contrast though, it has been revealed that one class of exonintron circular RNAs associated with RNA polymerase 
II in humans, promotes or increases the expression of their parental genes ( $\mathrm{Li}$ et al., 2015). Circular RNAs can also regulate the alternate splicing of transcripts (Lasda and Parker, 2014) and they can be sponges for RNA-binding proteins or ribonucleoprotein complexes (Hentze and Preiss, 2013). Finally, some circular RNAs can be bound by ribosomes and translated into functional proteins in vitro and in vivo (Wang and Wang, 2015; Legnini et al., 2017). Besides being translated themselves, some circular RNAs can also regulate the translation of their associated linear mRNAs and bind to a range of other proteins (Wilusz, 2018). Taken together, it can therefore be reasonably suggested that circular RNAs are worthy of further investigation and that their study may contribute to our understanding of the underlying mechanisms controlling complex and economically important traits, including the development of the mammary gland and milk production.

The mammary gland is a crucial organ in mammals and is involved in neonate survival, the acquisition of passive immunity, and early-life nutrition. Its development involves cyclical and dynamic periods of growth, differentiation, milk production, and regression; it underpins milk production for human consumption from domesticated species. Many studies have confirmed that the development of the mammary gland and lactation are coordinated activities, and that they are regulated by a large number of expressed genes and noncoding RNAs. However, compared with our knowledge of mammary gland mRNAs (Paten et al., 2015; Shi et al., 2015) and microRNAs (Li et al., 2012; Cai et al., 2017), little is known about the expression, characterization, and function of circular RNAs in the mammary gland.

There are very few reports about the activity of circular RNAs in the mammary gland, but in humans, $\mathrm{Xu}$ et al. (2017) found that the number of circular RNAs identified in mammary gland tissue $(9,665$ candidates), was higher than in the adrenal gland $(2,311$ candidates), the pancreas (1,791 candidates), and the thyroid (3,777 candidates). In rats, a total of 6,824 and 4,523 circular RNAs were found in the mammary gland at 2 different stages of lactation (Zhang et al., 2015), and in cattle, circular RNAs from 4 casein genes (CSN2, CSN3, CSN1S1, and CSN1S2) were reported to be highly expressed in bovine mammary gland, with 3 of these being expressed at a higher level on d 90 of lactation than on d 250 (Zhang et al., 2016).

There have been no reports on the presence or activity of circular RNAs in sheep mammary gland, with the exception of the study by Hao et al. (2020). They compared the expression levels of circular RNAs in lactating (20 d postpartum) mammary gland tissue from 2 breeds of sheep with different milk production performance: low lactation-yield Gansu Alpine Merino ewes and high lactation-yield Small Tail Han ewes. In contrast in this study, we investigate the circular RNA expression profile of mammary gland tissue from the same ewes at peak lactation and subsequently in the nonlactating period, using a high-throughput RNA sequencing (RNA-seq) approach. We describe the type, chromosome distribution, and length of these circular RNAs and undertake a Gene Ontology (GO) enrichment analysis of the parent genes of the differentially expressed circular RNAs. The linear RNA transcriptome of the same ovine mammary gland tissues as those used in the study has been previously described by Wang et al. (2020).

\section{MATERIALS AND METHODS}

\section{Sheep Investigated and RNA Preparation}

All experiments were performed in agreement with the care and use guidelines of experimental animals established by the Ministry of Science and Technology of the People's Republic of China (Approval number 2006-398), and the experimentation was also approved by Gansu Agricultural University, Lanzhou, China.

The mammary gland tissues used in the study were the same as those investigated by Wang et al. (2020). Briefly, 9 healthy, 3-yr-old Small Tail Han sheep obtained from the Jinzihe Sheep Breeding Company (Tianzhu County, China) were selected for the study. These sheep were all of the same parity (fourth parity) and had produced triplet lambs. Mammary gland biopsy tissues were first collected at peak lactation $(22 \mathrm{~d}$ postpartum) and a second biopsy sample was collected from the same 9 ewes $25 \mathrm{~d}$ after the cessation of lactation (when the ewes were not pregnant). The collected samples were immediately frozen in liquid nitrogen and then stored at $-80^{\circ} \mathrm{C}$ until RNA could be extracted.

Total RNA from the mammary gland tissues was isolated using TRIzol reagent (Invitrogen, Carlsbad, CA). The quantity and quality of the RNA was checked using a NanoDrop 8000 spectrophotometer (NanoDrop Technologies, Wilmington, DE) and the integrity of the RNA was assessed using an Agilent 2100 Bioanalyzer (Agilent Technologies, Santa Clara, CA). The RNA integrity number (RIN) derived from this technology was used to select high quality RNA samples (RIN >7), and rRNA was removed from these samples using a RiboMinus Kit (Invitrogen). The remaining mammary gland RNA was used for the following analyses.

\section{RNA Sequencing and Data Analysis}

To minimize the effect of individual ewe variation in mammary gland RNA, the RNA was pooled for RNA- 
seq, using an approach described by Paten et al. (2014). Briefly, equal volumes of the RNA extracted from the 9 nonlactating ewe mammary gland tissues were pooled into 3 groups of 3 randomly selected samples. Similarly, RNA from the 9 ewes at peak lactation were pooled into $3 \times 3$ randomly selected samples.

The pooled RNA samples were paired-end sequenced using an Illumina HiSeq 2500 sequencer (Illumina Inc., San Diego, CA) by the Shanghai Personal Biotechnology Company Ltd. (Shanghai, China). Clean reads were obtained by removing low-quality reads with quality scores $<$ Q20 (namely, the proportion of read bases whose error rate is less than $1 \%$ ) and removing the adapter reads in the raw reads. The clean reads were mapped to Oar_rambouillet_v1.0 using HISAT2. The software Find_circ (Memczak et al., 2013) was then used to identify the circular RNAs. Briefly, 20mers from both ends of these reads were extracted and aligned independently to the reference genome to find unique anchor positions within the splice sites. Anchor reads that mapped to the reversed (head-to-tail) orientation, represented circRNA splicing. Anchor alignment can be extended such that the original read sequence is completely aligned and the inferred breakpoints were flanked by GU/AG splice signals. Sequences with multiple mappings and ambiguous breakpoints were discarded. The type, chromosome distribution and length distribution of the circular RNAs identified, were statistically analyzed according to the results from the identification of find_circ and annotation of the reference Oar_rambouillet_v1.0.

The expression level of each annotated circular RNA was normalized using a reads per kilobase of transcript per million mapped reads (RPKM; a normalized unit of transcript expression) approach, and the differentially expressed (between the 2 periods of mammary gland development) circular RNAs were identified using the DEGseq R package (Wang et al., 2010) with the parameters of a $\mid$ fold-change $\mid>2.0$ and $P$-value $<0.05$ being set as the threshold for selecting the differentially expressed circular RNAs.

\section{Validation of Circular RNA Presence Using Reverse Transcriptase-PCR Amplification and DNA Sequencing}

Circular RNA has a head-to-tail junction that results in a structure that is different to comparable linear transcripts. Reverse transcription-PCR (RT-PCR) amplification can be used to validate the presence of these circular structures. Accordingly, aliquots $(2 \mu \mathrm{L})$ of the RNA samples that were used for the RNA-seq analysis, were also used as templates to synthesize cDNA using a RT-PCR kit (Takara, Dalian, China). The cDNA products were then amplified using PCR primers (Table 1) for circ_016906, circ_022214, ciRNA_022888, circ_018782, circ_011411, circ_017424, circ_021440, circ_014489, and circ_016842. The PCR products were checked for quality using agarose gel (1.0\%) electrophoresis and then sequenced using Sanger sequencing and the same PCR primers. The sequences of the PCR products were aligned to the sheep reference genome and RNA-seq data to confirm the location of the junction sites in the circular RNAs.

\section{Validation of the RNA-seq Results Using Quantitative RT-PCR Analyses}

To confirm the reliability of the RNA-seq results, 9 differentially expressed circular RNAs (circ_016906, circ_022214, ciRNA_022888, circ_018782, circ_011411, circ_017424, circ_021440, circ_014489, and circ_016842) that were the same as those analyzed using RT-PCR were subjected to quantitative RT-PCR (RT-qPCR). These also used the RNA samples extracted originally for the RNA-seq analysis. A cDNA was synthesized us-

Table 1. PCR primers used to amplify specific circular RNAs

\begin{tabular}{llll}
\hline CircRNA & Forward $\left(5^{\prime} \rightarrow 3^{\prime}\right)$ & Reverse $\left(5^{\prime} \rightarrow 3^{\prime}\right)$ & Amplicon size $(\mathrm{bp})$ \\
\hline circ_016906 & CCCTGTGAGAATGGCTGGAA & CCCTTAGGTGCCCACTTCC & 174 \\
circ_022214 & TCTCAGAGTCGGGCTTGTC & GTGATGCTCCACCTCTTCC & 131 \\
circ_022888 & CCCCAGATGTGAGCCTTGA & CTGGCACTTTCATTTCACCTTC & 186 \\
circ_018782 & CCTGGCGGTCAGCAAGAT & GCCTCGTACACCACCATCTCG & 188 \\
circ_011411 & AAACATTACCTGCCATCA & GTTTGAGCCTTTCCTTGC & 140 \\
circ_017424 & GGGACTCCCATTATTTCA & ATCCAGTTGAGCCCTTA & 170 \\
circ_021440 & CCTTCCCTCCTGTCAATA & CACCGTCTTTCCATTTCT & 115 \\
circ_014489 & TGCCACGTCTAGGTGAGCC & CCAGTCCCTCCTTGTCCC & 180 \\
circ_016842 & CCGAGTGGGTGTTTGTCT & GTGGCTCACTGGTTTGCT & 101 \\
$P R P F 3$ & ACAGATGATGGAAGCAGCAA & GGTTGGGAGGATGAAGGAGT & 116 \\
CUL1 & AAAAATACAACGCCCTGGTG & CTGAGCCATCTTGGTGACTG & \\
\hline
\end{tabular}


ing an RT-PCR kit (Takara, Dalian, China), and then RT-qPCR reactions were performed in triplicate using a SYBR qPCR Master Mix (Applied Biosystems, Foster City, CA) on an Applied Biosystems QuantStudio 6 Flex (Thermo Lifetech, Waltham, MA) platform. The genes PRPF3 and CUL1 were used as reference genes for standardization as suggested by Paten et al. (2014). The primers used for the RT-qPCR were the same as those used for the RT-PCR (Table 1) and the relative expression levels were calculated using a $2^{-\Delta \Delta \mathrm{Ct}}$ method (Livak and Schmittgen, 2001).

\section{Gene Ontology Enrichment Analysis and Target MicroRNA Predictions}

Gene Ontology enrichment analysis was used to investigate the main functions of the parent genes of the differentially expressed circular RNAs using the DAVID tool (https://david.ncifcrf.gov). The target microRNAs of 5 of the differentially expressed circular RNAs were predicted using the miRanda algorithm (Miranda et al., 2006) and the interaction network of the circular RNAs and their target microRNAs was analyzed using starBase and then drawn using Cytoscape (Smoot et al., 2011).

\section{RESULTS}

\section{Identification of Circular RNAs in the Ovine Mammary Gland Tissue}

A total of $109,543,510$ and $107,562,553$ clean reads that could be mapped to the ovine genome assembly Oar_rambouillet_v1.0 were obtained from mammary gland tissue at peak lactation and during the nonlactating period, respectively. Circular RNAs were detected in mammary gland tissue at both peak lactation $(\mathrm{n}=$ $3,278)$ and in the nonlactating period $(\mathrm{n}=1,756)$. Of all the circular RNAs detected, 1,108 circular RNAs $(28.22 \%)$ were expressed in both periods. Most notably, all 4 casein-coding genes (CSN2, CSN1S1, CSN1S2, and $C S N 3)$ and 2 whey protein-coding genes (BLG and $L A L B A$ ), produced circular RNAs in the ovine mammary gland tissue at peak lactation.

\section{Authentication of the Sheep Mammary Gland Tissue Circular RNAs}

To validate the authenticity of the circular RNAs identified, RT-PCR amplification and DNA sequencing were performed on 9 selected circular RNAs. The RTPCR results revealed that all 9 circular RNAs could be detected and that they produced a band on agarose gel electrophoresis of the expected size (Figure 1a). DNA sequencing confirmed the presence of head-to-tail splice junctions as suggested by the RNA-seq analyses (Figure $1 \mathrm{~b}$ ) and the size of the circular RNAs.

\section{Characterization of Circular RNAs in the Ovine Mammary Gland Tissue}

Of the 6 circular RNAs types identified based on the mapping of the circular RNAs to the sheep genome (Oar_rambouillet_v1.0), annotated exons (annot_exons) were the most common sequences identified, accounting for 80.9 and $69.9 \%$ of the circular RNAs in the peak lactation and nonlactating mammary gland tissue respectively. This was followed by one_exon (6.1 and $8.3 \%$ ) circular RNAs. The remaining circular RNAs were smaller amounts of intron_exon, intergenic, antisense and intron (Figure 2a) sequences. The circular RNAs identified in the study were widely distributed across all the ovine chromosomes, including the X chromosome, but the greatest number were concentrated on chromosomes 1 to 3 (Figure $2 \mathrm{~b}$ ). The length of the majority of circular RNAs was less than $1 \mathrm{~kb}$ (Figure 2c).

\section{Analysis of Differentially Expressed Circular RNAs and GO Enrichment Analysis of the Parent Genes}

Forty-one differentially expressed circular RNAs were identified in comparing mammary gland tissue at peak lactation with mammary gland tissue from the nonlactating period. These included 40 upregulated circular RNAs and one downregulated circular RNA in the mammary gland tissue at peak lactation (Table 2). Of all the upregulated circular RNAs in the mammary gland tissue at peak lactation, 2, one and one circular RNAs were derived from $B L G, C S N 2$ and $L A L B A$, respectively.

To validate the reliability of our RNA-seq results, 8 upregulated circular RNAs and one downregulated circular RNA in the peak-lactating samples, were selected and subjected to RT-qPCR analysis. These were the same circular RNAs as those used for the RTPCR validation (i.e., $B L G$ for circ_016906, ATP2C2 for circ_022214, AHNAK for ciRNA_022888, COPG1 for circ_018782, SLC39A8 for circ_011411, CSN2 for circ_017424, ENSOARG00000007064 for circ_021440, $B L G$ for circ_014489 and ATP2C2 for circ_016842). The results from the RT-qPCR were consistent with those obtained from the RNA-seq analysis. For example, circ_017424 and circ_014489 were only expressed 
A

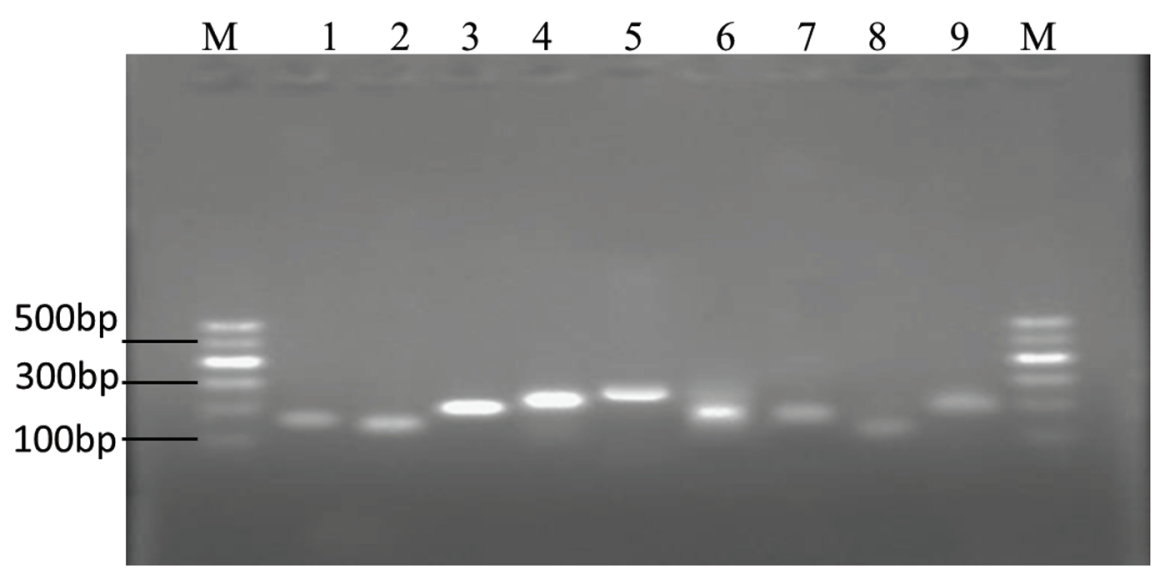

B
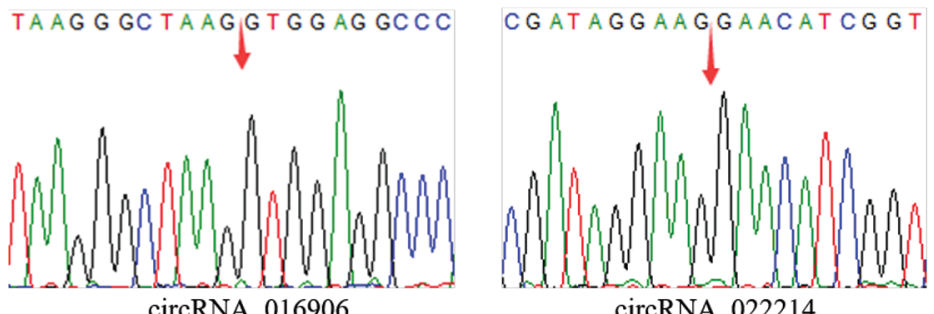

A A GC TCA AG G G C C C TAAG T T circRNA 022214
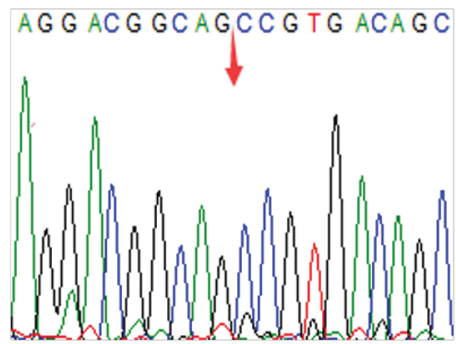

circRNA_018782

AC G TA T CA A G C TA T G G AG AC
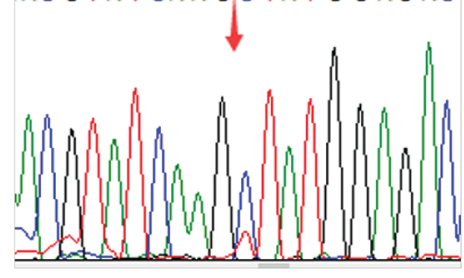

circRNA 021440
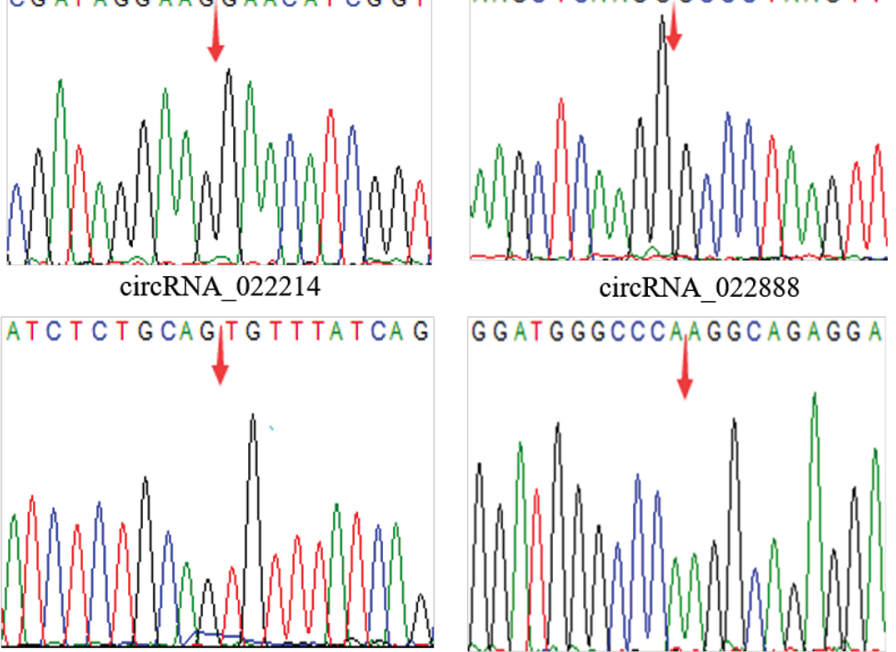

circRNA_011411

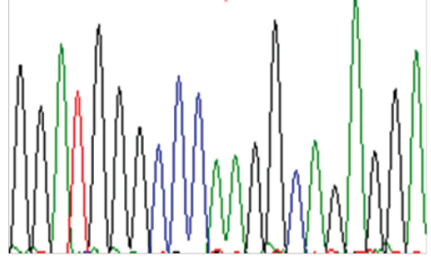

circRNA_017424

T G C C TC T G G GG GCCCG G G AG
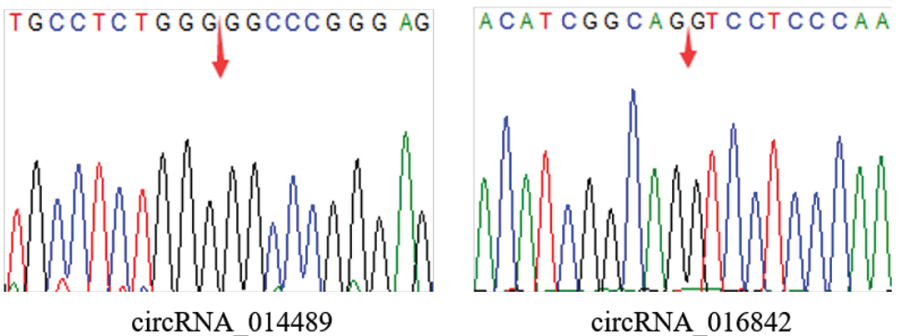

Figure 1. Validation of the presence of selected circular RNAs in ovine mammary gland tissue. (A) Reverse transcriptase-polymerase chain reaction (RT-PCR) amplimers derived from the circular RNAs using divergent primers for ovine mammary gland RNA. M: Marker; 1: circRNA_016906; 2: circRNA_022214; 3: circRNA_022888; 4: circRNA_018782; 5: circRNA_011411; 6: circRNA_017424; 7: circRNA_021440; 8: circRNA_014489; 9. circRNA_016842. (B) The head-to-tail splice junctions for the circRNAs were confirmed by DNA sequencing and are marked with a red arrow on the DNA sequence chromatograms.

in the mammary gland tissue at peak lactation. As the $\log _{2}$ fold-change for peak lactation relative to the nonlactating period was infinity for these 2 circular RNAs, their relative expression level are not shown in Figure 3.

The expression level of circRNA_022888 was lower in the mammary gland tissue samples at peak lactation than in the nonlactating period mammary gland tissue samples, whereas the expression level of circ_016906, circ_022214, circ_018782, circ_011411, circ_021440, and circ_016842 was higher in the mammary gland tissue samples at peak lactation (Figure 3). These results suggest that the RNA-seq results are reliable and that 
the circular RNAs identified in the study, reflect differential expression in vivo in mammary gland tissue that was at different stages of development.
To further understand the potential functional pathways of the differentially expressed circular RNAs between the 2 stages of mammary gland development,

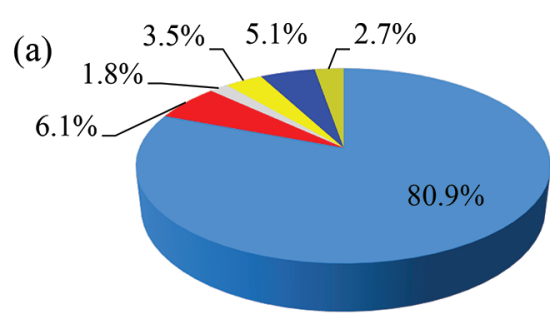

Peak lactation

(b)

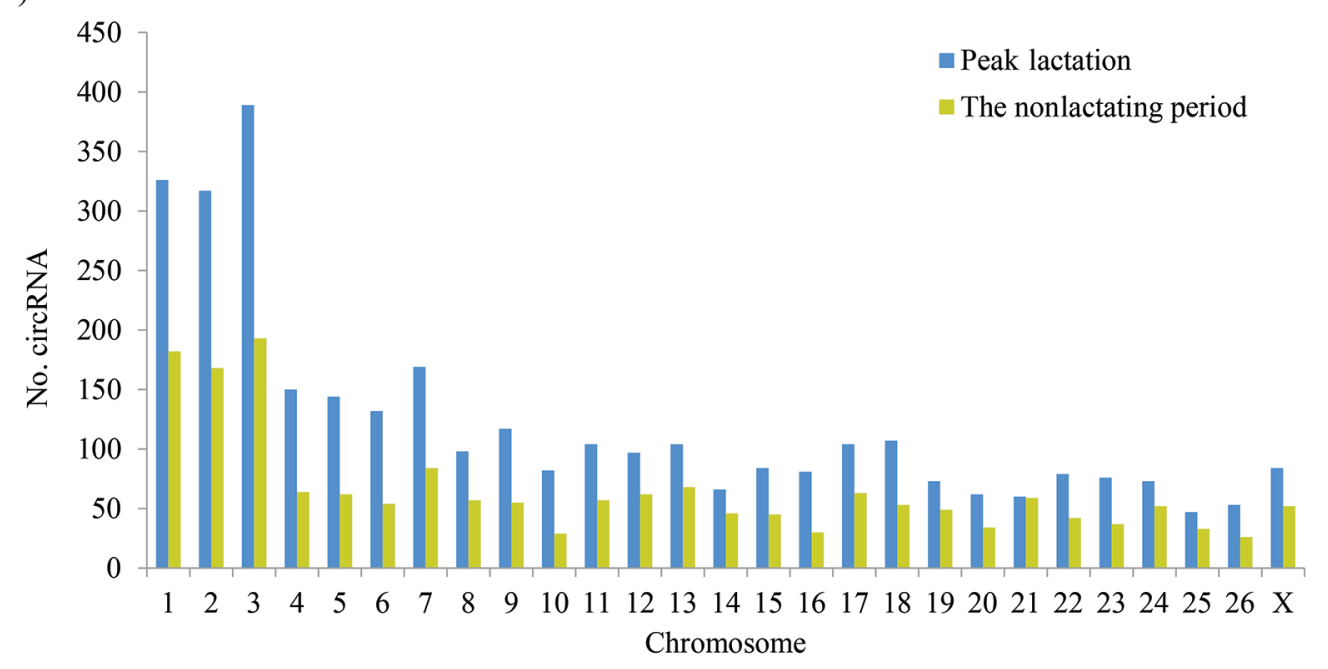

(c)

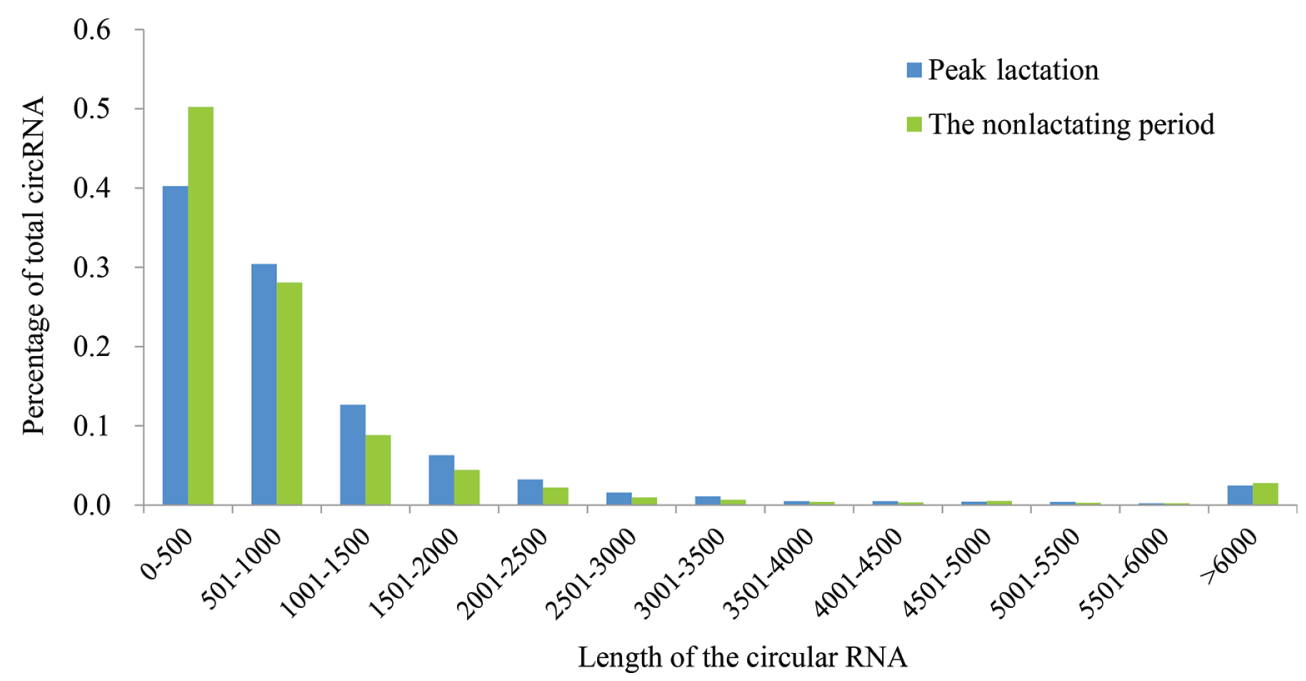

Figure 2. General characteristics of the circular RNAs in the ovine mammary gland tissue. (a) The circular RNA types in the peak lactation mammary gland tissue and the nonlactating period mammary gland tissue. (b) The chromosomal location of origin of the circular RNAs in the ovine mammary gland tissue. (c) The length distribution of the circular RNAs from the ovine mammary gland tissue. 
a GO enrichment analysis of the parental genes of the differentially expressed circular RNAs was performed. According to 3 classifications of GO, the most enriched biological process (GO-BP) terms were biological_process, single-organism process, cellular process, singleorganism cellular process, and biological regulation; the most enriched cell component (GO-CC) terms were membrane, membrane-bounded organelle, organelle, cell, and cell part; and the most enriched molecular function (GO-MF) terms were molecular_function, binding, protein binding, ATP binding, and ion binding (Figure 4).

\section{The Target MicroRNAs of the Differentially Expressed Circular RNAs}

The results from the miRanda analysis revealed a total of 1,423 target microRNAs identified to the 41 differentially expressed circular RNAs. Of the 41 differentially expressed circular RNAs, 4 upregulated

Table 2. Differentially expressed circular RNAs from a comparison of mammary gland tissue at peak lactation and from during the nonlactating period in sheep ${ }^{1}$

\begin{tabular}{|c|c|c|c|c|c|c|c|}
\hline ID & Gene name & $\begin{array}{c}\text { BaseMean } \\
\text { Peak }^{2}\end{array}$ & $\begin{array}{c}\text { BaseMean } \\
\text { Non }^{3}\end{array}$ & $\begin{array}{c}\text { Fold } \\
\text { change }\end{array}$ & $\begin{array}{c}\log _{2} \text { fold } \\
\text { change }\end{array}$ & $P$-value & Change $^{4}$ \\
\hline circ_022214 & ATР2C2 & 789.12 & 0.93 & 848.52 & 9.73 & 7.89E-19 & Upregulated \\
\hline circ_011411 & SLC39A8 & 656.39 & 5.23 & 125.50 & 6.97 & $7.18 \mathrm{E}-09$ & Upregulated \\
\hline circ_016906 & $B L G$ & 316.56 & 1.56 & 202.92 & 7.66 & $1.29 \mathrm{E}-05$ & Upregulated \\
\hline circ_021440 & ENSOARG00000007064 & 289.32 & 3.23 & 89.57 & 6.48 & 0.0001 & Upregulated \\
\hline circ_014121 & $O B P 2 A$ & 103.15 & 0 & Inf & Inf & 0.0002 & Upregulated \\
\hline circ_016842 & АТР2C2 & 136.55 & 0.38 & 359.34 & 8.49 & 0.0003 & Upregulated \\
\hline circ_008383 & Intergenic & 73.58 & 0 & Inf & Inf & 0.0015 & Upregulated \\
\hline circ_014489 & $B L G$ & 65.36 & 0 & Inf & Inf & 0.0016 & Upregulated \\
\hline circ_022150 & Metazoa_SRP & 557.16 & 125.53 & 4.44 & 2.15 & 0.0056 & Upregulated \\
\hline circ_023810 & ENSOARG00000001834 & 53.29 & 0 & Inf & Inf & 0.0078 & Upregulated \\
\hline circ_008952 & $L A L B A$ & 49.35 & 0 & Inf & $\operatorname{Inf}$ & 0.0146 & Upregulated \\
\hline circ_018782 & COPG1 & 369.33 & 66.52 & 5.55 & 2.47 & 0.0153 & Upregulated \\
\hline circ_024119 & HIPKЗ & 46.50 & 0 & Inf & $\operatorname{Inf}$ & 0.0173 & Upregulated \\
\hline circ_017031 & $M F S D 6$ & 39.43 & 0 & Inf & Inf & 0.0185 & Upregulated \\
\hline circ_009591 & $M F S D 6$ & 37.43 & 0 & $\operatorname{Inf}$ & Inf & 0.0185 & Upregulated \\
\hline circ_012459 & $C O B L L 1$ & 37.45 & 0 & Inf & Inf & 0.0191 & Upregulated \\
\hline circ_000902 & WHSC1 & 34.69 & 0 & $\operatorname{Inf}$ & Inf & 0.0198 & Upregulated \\
\hline circ_001211 & $C D H 1$ & 33.13 & 0 & $\operatorname{Inf}$ & $\operatorname{Inf}$ & 0.0199 & Upregulated \\
\hline circ_017424 & $C S N 2$ & 32.15 & 0 & $\operatorname{Inf}$ & $\operatorname{Inf}$ & 0.0201 & Upregulated \\
\hline circ_001879 & $X D H$ & 31.77 & 0 & Inf & $\operatorname{Inf}$ & 0.0204 & Upregulated \\
\hline circ_002662 & MID2 & 28.24 & 0 & Inf & Inf & 0.0212 & Upregulated \\
\hline circ_010404 & Intergenic & 27.23 & 0 & $\operatorname{Inf}$ & Inf & 0.0215 & Upregulated \\
\hline circ_005803 & SLC20A2 & 26.13 & 0 & $\operatorname{Inf}$ & Inf & 0.0216 & Upregulated \\
\hline circ_015064 & $L O N P 2$ & 25.21 & 0 & $\operatorname{Inf}$ & Inf & 0.0226 & Upregulated \\
\hline circ_017249 & SLC39A8 & 25.30 & 0 & $\operatorname{Inf}$ & $\operatorname{Inf}$ & 0.0228 & Upregulated \\
\hline circ_021892 & $M P P 6$ & 25.47 & 0 & $\operatorname{Inf}$ & Inf & 0.0233 & Upregulated \\
\hline circ_011582 & $T C 2 N$ & 22.16 & 0 & $\operatorname{Inf}$ & Inf & 0.0252 & Upregulated \\
\hline circ_017782 & $P R R C 1$ & 21.79 & 0 & Inf & Inf & 0.0261 & Upregulated \\
\hline circ_002288 & FОХОЗ & 20.69 & 0 & Inf & Inf & 0.0265 & Upregulated \\
\hline circ_015322 & KIAA1324 & 20.28 & 0 & $\operatorname{Inf}$ & $\operatorname{Inf}$ & 0.0267 & Upregulated \\
\hline circ_006972 & $A C A C A$ & 20.43 & 0 & Inf & $\operatorname{Inf}$ & 0.0282 & Upregulated \\
\hline circ_020783 & FCHSD2 & 20.27 & 0 & Inf & Inf & 0.0300 & Upregulated \\
\hline circ_001091 & РPAР2A & 19.56 & 0 & $\operatorname{Inf}$ & $\operatorname{Inf}$ & 0.0341 & Upregulated \\
\hline circ__ 002602 & $A B C G 2$ & 17.50 & 0 & $\operatorname{Inf}$ & Inf & 0.0352 & Upregulated \\
\hline circ_ 014277 & $K A T 2 B$ & 15.65 & 0 & $\operatorname{Inf}$ & Inf & 0.0385 & Upregulated \\
\hline circ_015262 & TNFRSF21 & 11.95 & 0 & $\operatorname{Inf}$ & Inf & 0.0403 & Upregulated \\
\hline circ_002235 & SEMA $3 A$ & 11.13 & 0 & $\operatorname{Inf}$ & Inf & 0.0419 & Upregulated \\
\hline circ_002931 & $P R K A A 1$ & 11.08 & 0 & $\operatorname{Inf}$ & Inf & 0.0420 & Upregulated \\
\hline circ_023779 & PLIN2 & 10.38 & 0 & $\operatorname{Inf}$ & Inf & 0.0436 & Upregulated \\
\hline circ_ 006393 & $M A P 2 K_{4}$ & 10.28 & 0 & Inf & Inf & 0.0463 & Upregulated \\
\hline circ_022888 & $A H N A K$ & 82.59 & 432.15 & 0.19 & -2.39 & 0.0166 & Downregulated \\
\hline
\end{tabular}

${ }^{1}$ Inf $=$ infinity.

${ }^{2}$ BaseMean is used to homogenize the expression of the gene in both the total sample and the single sample in the DEGSeq R package (Wang et al., 2010). BaseMean Peak is the BaseMean value at peak lactation.

${ }^{3}$ BaseMean Non is the BaseMean value in the nonlactating period.

${ }^{4}$ The difference in the expression of a specific circular RNA in the peak lactation mammary gland tissue, compared with the nonlactating period mammary gland tissue. 


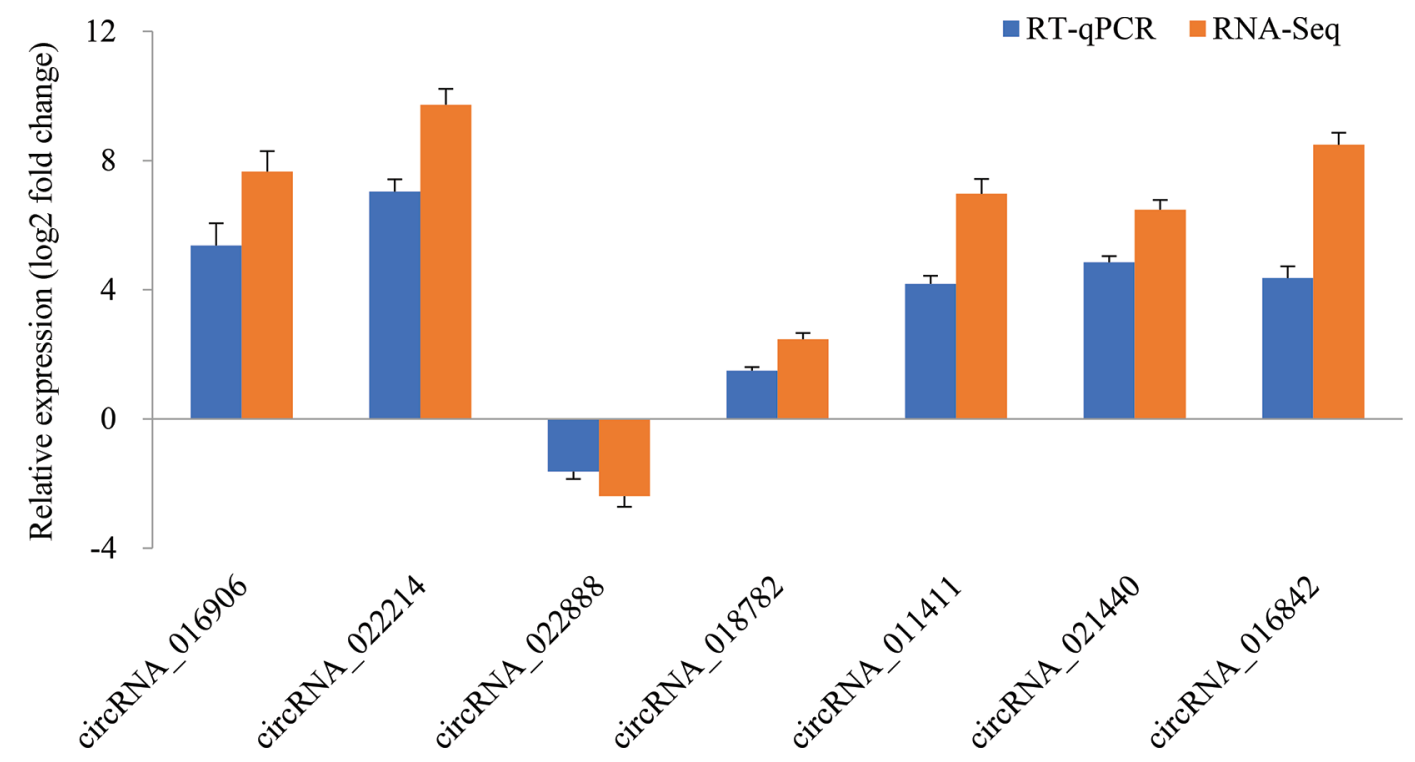

Figure 3. A comparison of circular RNA levels using the RNA-seq results and the quantitative reverse transcriptase-polymerase chain reaction (RT-qPCR) results for 7 differentially expressed circular RNAs. Three independent replicates were used for each sample, and error bars illustrate SD of the means.

circular RNAs and 1 downregulated circular RNA in the mammary gland tissue at peak lactation were then selected to study the interaction of the circular RNAs and their target microRNAs. These included the only downregulated circular RNA (circ_022888), the most common upregulated circular RNA (circ_022214), 2 circular RNAs derived from $B L G$ (circ_016906 and circ_014489), and one circular RNA derived from LALBA (circ_008952).

There were 456 target microRNAs in total for these 5 circular RNAs, ranging from 5 target microRNAs for circ_022214 to 152 targets for circ_008952. For each circular RNA, the target microRNAs with the largest total score and absolute value of total energy were selected from the 456 target microRNAs, and a circular RNA-microRNA interaction network was constructed (Figure 5). Some microRNAs that have been previously reported to be associated with mammary gland development and lactation were identified in this analysis, including miR-200a, miR-200b, miR-21, miR-181a, miR-107, miR-23a, miR-23b, miR-103, and miR-362 (Rogler et al., 2009; Finnerty et al., 2010; Li et al., 2012; Galio et al., 2013).

\section{DISCUSSION}

As a consequence of technical limitations and the low expression level of most circular RNAs, few circular RNAs were found in eukaryotes until the appearance of RNA sequencing technologies such as RNA-seq. Stud- ies have now demonstrated that RNA-seq technology is powerful and capable of accurately identifying and characterizing circular RNAs, and recently thousands of unique circular RNAs have been annotated in a variety of species based on the use of RNA-seq.

Measurement noise has been a challenge in analyzing RNA-seq data. Therefore, RT-qPCR has been used to validate the occurrence of circular RNAs originating from RNA-seq in sheep and cattle due to its high sensitivity and accuracy ( $\mathrm{Li}$ et al., 2017a, b; Gao et al., 2018; Hao et al., 2020). Although a good overall agreement for relative expression levels has been found between RT-qPCR and RNA-seq, some differences in expression level measurements can also be observed (SEQC/MAQC-III Consortium, 2014). Defining common trends in expression between different samples has therefore become an accepted way of validating RNAseq results with RT-qPCR, and our results support this approach (Figure 3).

In the study, 2 periods of mammary gland development were selected, with the nonlactating ewes being sampled on d 25 after the cessation of lactation. The nonlactation period investigated in the study therefore coincides with involution of the mammary gland, as Tatarczuch et al. (1997) found that the ovine mammary gland was completely involuted by $30 \mathrm{~d}$ after weaning in sheep.

The pooling of samples in biomedical studies has now become a common practice. For example, $>15 \%$ of the data sets deposited in the Gene Expression Omnibus 

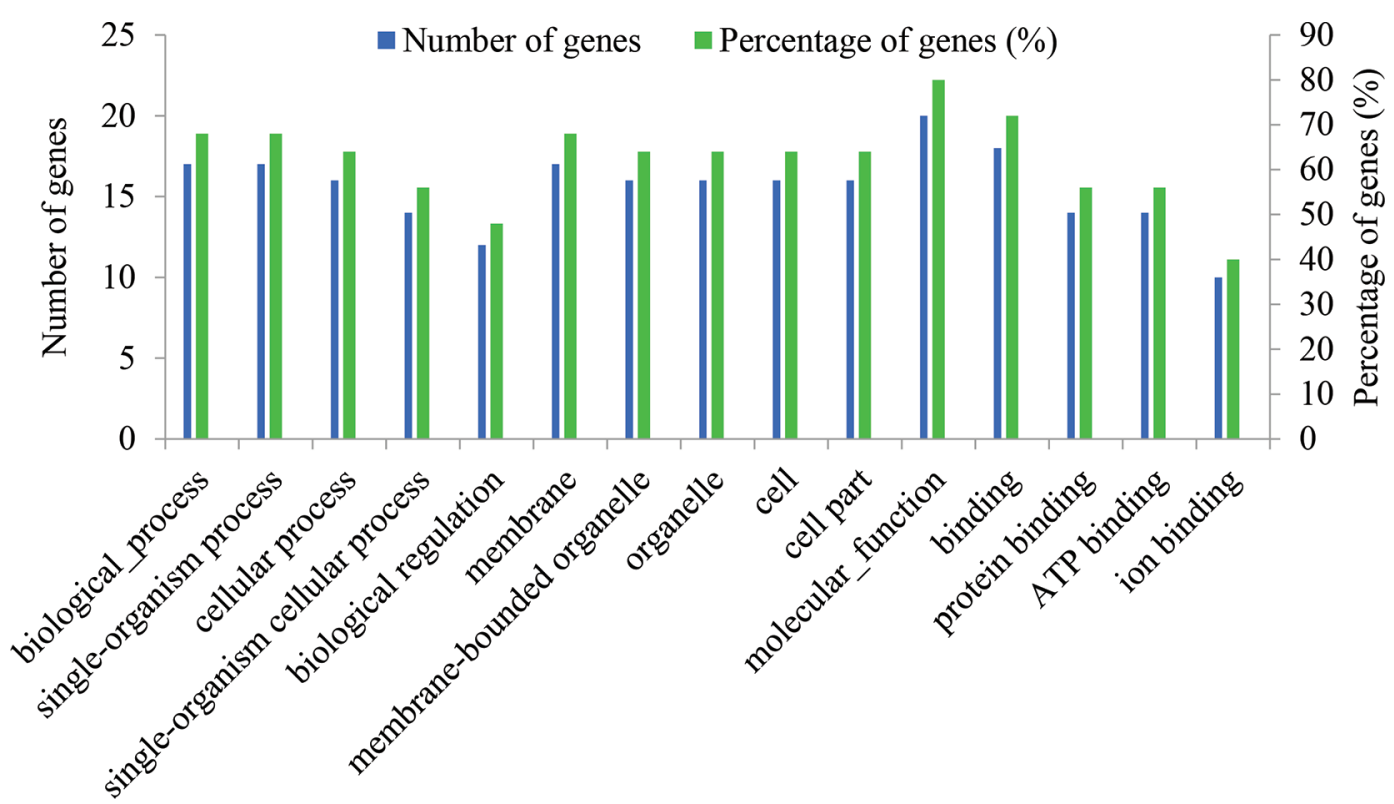

Figure 4. Most common Gene Ontology terms of the parental genes from which the differentially expressed circular RNAs were derived, when comparing the mammary gland tissue from ewes at peak lactation and in the nonlactating period.

database involved pooled RNA samples (Kendziorski et al., 2005). Kendziorski et al. (2005) also acknowledged that inference for most genes was not adversely affected by pooling and thought that pooling is beneficial when many subjects are pooled, provided that independent samples contribute to multiple pools. Paten et al. (2014, 2015) also justify the use of pooling and observed that pooling can accommodate the variation that can occur between individual samples in ovine mammary gland tissues. Our approach was planned based on the Paten et al. (2014) and Paten et al. (2015) studies and this means our comparison would be between an average expression profile for the 2 periods.

However, caution is needed in using this approach, as Rajkumar et al. (2015) found lower positive predictive value for the differentially expressed genes in pooled samples. In this study, interindividual variation may be quite important in discerning between differentially

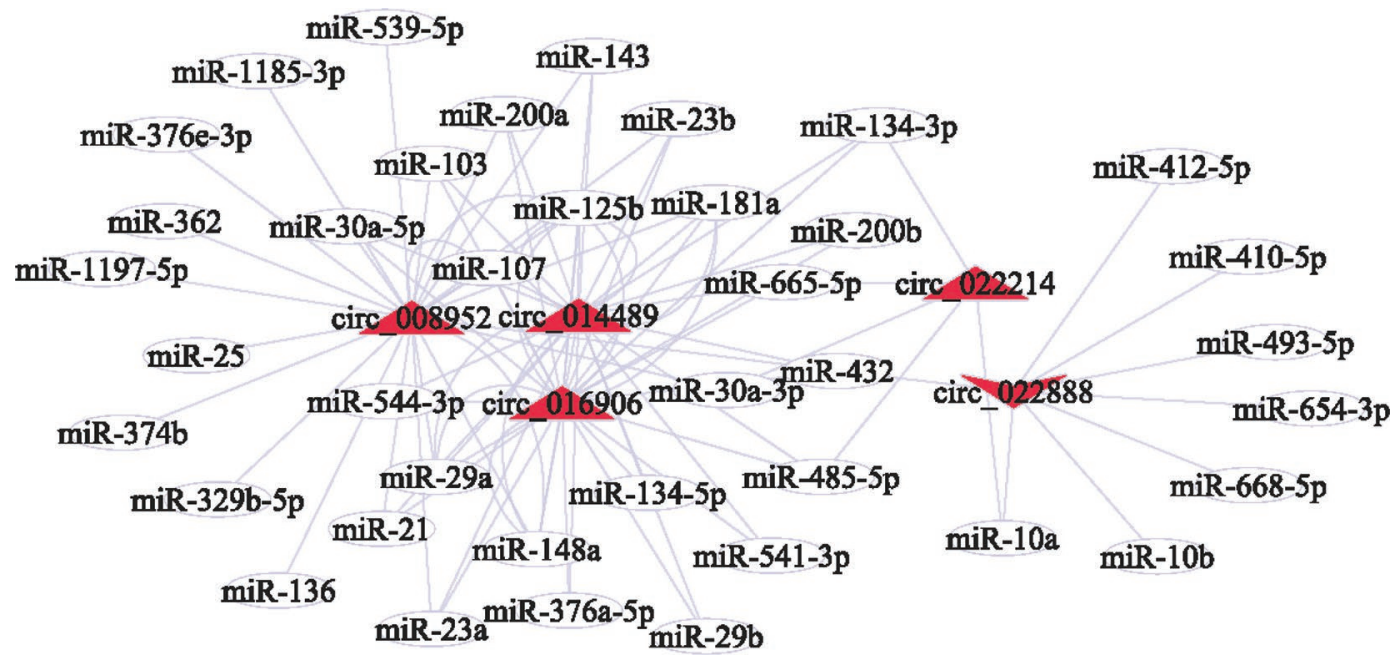

Figure 5. The circular RNA-microRNA interaction network. The triangles and inverted triangle represent upregulated and downregulated circular RNAs in the mammary gland tissue from ewes at peak lactation versus nonlactating ewes, respectively. The gray circles represent the predicted target microRNAs for the 5 differentially expressed circular RNAs. 
expressed circular RNAs that occur because of causal effects, and the presence of differentially expressed circular RNAs that occur as a consequence of individual-specific environmental effects. In this context, the pooling of samples may have limited the study, as it is impossible to easily distinguish between coincidental environmental effects and causal effects.

In our study, 3,278 and 1,756 circular RNAs were identified and annotated in mammary gland tissue from sheep at peak lactation and in the nonlactating period, respectively. Only 1,108 of these circular RNAs were expressed at both stages of mammary gland development. The number of circular RNAs identified at peak lactation in this study was very similar to what was described by Hao et al. (2020), who described an average of 4,020 circular RNAs in ovine mammary gland at lactation (20 d postpartum) from low lactation-yield Gansu Alpine Merino ewes and high lactation-yield Small Tail Han ewes. Additionally, in cattle mammary glands, there were 4,804 and 4,048 distinct circular RNAs identified on d 90 and d 250 postpartum, respectively (Zhang et al., 2016). The number of circular RNAs we identified in the nonlactating mammary gland tissue could not be compared, as there is an absence of relevant studies for comparison.

Compared with circular RNA presence in other tissues from sheep, the total number of circular RNAs identified here was less than has been described for muscle and pituitary gland, with 6,113 and 10,226 circular RNAs being identified respectively ( $\mathrm{Li}$ et al., 2017a, b). This likely reflects tissue-specific expression patterns, and the unique character of the different tissues. In this respect, differential tissue-specific expression of circular RNAs has also been reported in humans (Rybak-Wolf et al., 2015) and pigs (Huang et al., 2018).

Our observation that the majority of circular RNAs detected mapped to protein-coding exons, is consistent with findings in dairy cows (Zhang et al., 2016), other cattle (Gao et al., 2018), and humans (Guo et al., 2014). Other types of circular RNAs that have been identified in humans (Memczak et al., 2013) were also found in the study, including intergenic, antisense, intron_exon, and intronic circular RNAs, and these circular RNAs have been described in mammary gland tissues from sows (Sun et al., 2020), dairy cows (Zhang et al., 2016), and rats (Zhang et al., 2015). For example, intergenic circular RNAs accounted for 5.2 and $3.0 \%$ of all circular RNAs in lactating sows (Sun et al., 2020) and dairy cows (Zhang et al., 2016), respectively. It is noteworthy that of annot_exons circular RNAs identified in the study, the majority of circular RNAs consisted of exons from a unique gene, whereas 63 of circular RNAs were found to include multiple exons that originated from 2 genes. Why circular RNAs from 2 different genes occur and what roles they may play are not clear, and they need to be studied in greater detail in future. Most the circular RNAs detected in the sheep mammary gland were short, being less than $1 \mathrm{~kb}$ in length, and this is also consistent with results from the analysis of circular RNA from the mammary gland of dairy cows (Zhang et al., 2016) and testis of cattle (Gao et al., 2018). Ovine chromosomes 1 to 3 produced the most circular RNAs in this study, which is perhaps unsurprising as these chromosomes are the largest in size, and comprise approximately $28.8 \%$ of the total length of the genome (Oar_rambouillet_v1.0; https://www.ncbi.nlm.nih .gov/genome/?term = Oar_rambouillet_v1.0).

It was noteworthy that 4 milk casein-encoding genes (CSN2, CSN1S1, CSN1S2, and CSN3) and 2 milk whey protein-encoding genes ( $B L G$ and $L A L B A)$ produced circular RNAs in the peak lactation mammary gland tissue. In the mammary gland of dairy cattle, it was also found that all 4 milk casein-coding genes produced circular RNAs (Zhang et al., 2016). In a previous study of ovine mammary gland transcriptome at peak lactation (20 d postpartum) from low lactation-yield Gansu Alpine Merino ewes and high lactation-yield Small Tail Han ewes (Hao et al., 2019), CSN2 was found to be the most abundant gene expressed, with the highest RPKM value at peak lactation in the mammary gland tissue. However, in this study, and unlike the other 5 genes, CSN2 only produced one circular RNA (circ_017424). This is perhaps not surprising as Zhang et al. (2016) also only detected a single CSN2 circular RNA in the mammary gland of dairy cows.

The circular RNAs circ_016906 and circ_014489 both originated from $B L G$ and the same 133 target microRNAs were identified for both. The presence of both of these circular RNAs was confirmed by RT-PCR and sequencing and the latter analysis confirmed they were unique circular RNAs. By aligning the sequences of the 2 circular RNAs to ovine $B L G$ (GenBank accession no. X12817.1; Harris et al.,1988), it was found that the 2 circular RNAs included the entirety of exon 1, exon 2 , exon 3, and exon 5, and part of exon 4 and the promoter, with the difference being that circ_016906 also included part of intron 6 . This difference in sequence between circ_016906 and circ_014489 may result in them occupying different cell locations and having different functions.

It has been suggested that circular RNAs containing intronic sequences are mainly located in the nucleus and are involved in the regulation of expression of their parent genes (Zhang et al., 2013). For example, Li et al. (2015) described how intron_exon circular RNAs can have a retained intron that allows them to interact 
with U1 snRNP to form a complex. The intron_exon circular RNA-U1 snRNP complexes can then further interact with the polymerase II transcription complex located at the promoters of their parent genes to enhance gene expression. Intron_exon circular RNAs may also regulate the expression of the parental genes transcriptionally to increase levels of both the circular RNA and the mRNA (Li et al., 2015). This may explain why in our findings circ_016906 had a 5.72-fold higher level of expression than circ_014489 in the peak lactation mammary gland tissue.

Exon-only circular RNAs are primarily located in the cytoplasm and can function as microRNA sponges (Zhang et al., 2013). The microRNAs are $\sim 21$-nt noncoding RNAs that inhibit protein synthesis by repressing translation, or by promoting the degradation of their target mRNAs (Carrington and Ambros, 2003). Increasingly evidence suggests that the circular RNAs thereby inhibit or relieve the repression of target mRNAs by microRNAs, and that this is a general phenomenon (Hansen et al., 2013).

Of the 40 upregulated circular RNAs in the mammary gland tissue at peak lactation, $83 \%$ (i.e., 33/40) were only expressed in the peak lactation mammary gland tissue and not in the nonlactating tissue. These included the CSN2 circular RNA (circ_017424), $L A L$ $B A$ circular RNA (circ_008952), BLG circular RNA (circ_014489), PPAP2A circular RNA (circ_001091), and PLIN2 circular RNA (circ_023779). The expression of specific circular RNAs at this stage of mammary gland development is consistent with the parent genes of these circular RNAs being involved in the synthesis and secretion of proteins and lipids in milk, with, for example, CSN2, $L A L B A$, and $B L G$ producing key protein components ovine milk (Suárez-Vega et al., 2016). Additionally, the protein encoded by PPAPQA belongs to the phosphatidic acid phosphatase family and plays a role in the synthesis of glycerolipids, which can transform phosphatidic acid into diacylglycerol (Tang et al., 2015). The protein PLIN2 is a member of the perilipin family of lipid droplet proteins and plays a role in both the formation and secretion of milk lipids (Chong et al., 2011).

Compared with the elevated expression levels of mRNAs in the mammary gland transcriptome analysis of Wang et al. (2020), the expression levels of the circular RNAs were comparatively low. For example, that study reported that the RPKM value of CSN2, $B L G$, and $L A L B A$ was $25,919,17,171$, and 11,234 respectively, in mammary gland tissue at peak lactation. However, the circular RNAs identified here that corresponded to these gene transcripts were 317 (circ_017424), 423 (circ_014489) to 792 (circ_016906), and 215 (circ_008952), respectively. It was further found that the total RPKM values of all the circular RNAs identified in the study only accounted for 0.2 to $0.3 \%$ of the mRNA, when comparing total RPKM for the circular RNAs with those for the mRNAs (Wang et al., 2020). This is consistent with the findings of Guo et al. (2014) in humans, where it was also observed that the majority of circular RNAs were expressed at lower levels than their canonical linear mRNAs. How such low levels of expression can have a physiological effect on mammary gland development is a mystery, but one possible explanation may be that these circular RNAs can either be involved in a catalytic role, or interact with other molecules that have important functions (Guo et al., 2014). Li et al. (2015) suggested that for the cis-effect of circular RNAs on their parent genes, the abundance of individual circular RNAs does not necessarily need to be high for the effect to occur.

In this study, most of the differentially expressed circular RNAs exhibited a similar direction of change in expression (up or down), when compared with their parent genes as described by Wang et al. (2020). For example, the $A H N A K$ transcript, which corresponded to the only downregulated circular RNA (circ_022888) in this study, was revealed by Wang et al. (2020) to have a lower level of expression in the mammary gland at peak lactation. The parent genes corresponding to 40 upregulated circular RNAs described here, were also expressed at higher levels in peak lactation mammary gland tissue than in nonlactating mammary gland tissue (Wang et al., 2020). Taken together, this would suggest that these differentially expressed circular RNAs and their parent genes may play similar roles in both the nonlactating and lactation periods.

In comparing the expression levels of some of the circular RNAs reported, with the parent genes (as described by Wang et al. (2020)), a positive relationship is observed for some genes, but not for others. For example, the expression level of circ_016906 had a strong positive correlation $(\mathrm{r}=0.836)$ with its parent gene $B L G$ for 3 pooled samples at peak lactation period. A connection between circular RNA levels and the level of expression of the parent genes in mammary gland tissue was also described by Zhang et al. (2016). In contrast, for circ_015322 in this study (average BaseMean 20), there was no obvious relationship in expression of 3 samples with its parent gene KIAA1324 (average BaseMean 10,472) in Wang et al. (2020) at peak lactation period. The failure to find a relationship may be a consequence of the circular RNA acting as a microRNA sponge, and not necessarily because of activity associated with the parent gene. Wilusz (2018) also suggested that there is often no clear correlation 
between expression levels of circular RNAs and their corresponding mRNAs. Taken together this suggests that the relationship between circular RNAs and their parent genes is complex.

The GO analyses revealed the main functions of the parent genes of the differentially expressed circular RNAs were related to molecular function, binding, protein binding, ATP binding, and ion binding in biological_process, single-organism process, cellular process, and biological regulation in cells, especially in organelles. This is consistent with the most enriched GO categories for the target genes of microRNAs in the mammary gland of lactating Laoshan dairy goats, these being associated with cellular process and biological regulation in the GO-BP, cell, cell part, organelle, and membrane-bounded organelle in the GO-CC, and binding and ion binding in the GO-MF (Ji et al., 2012). Dai et al. (2018) found the upregulated genes were significantly enriched in membrane and organelle processes in mammary gland during lactation compared with the dry period for Holstein dairy cows. They suggested that these biological processes and molecular functions were important for lactation or mammary gland development in domestic animals, given the observation that milk proteins are synthesized in an organelle (the endoplasmic reticulum) and that their production is an energetically costly process.

Some single circular RNA appeared to target a range of different microRNAs, whereas other circular RNAs only appear to interact with a single target microRNA. For example, circ_008952 could potentially interact with 152 target microRNAs, whereas circ_016906, circ_014489 and circ_008952 appear to only potentially interact with a single target microRNA. This finding is consistent with a previous study of muscle tissue gene expression in sheep (Li et al., 2017b), with them reporting that one circular RNA (circ_0000385) could potentially target 93 microRNAs, whereas circ_000385 and circ_0000582 only appeared to interact with miR-107.

Of the target microRNAs identified in the study, some had previously been reported to be associated with mammary gland development and lactation. For example, miR-107 has been reported to be involved in the metabolism of cellular lipids and thus the regulation of lactation (Finnerty et al., 2010; Li et al., 2012). The microRNA miR-23b may be involved in the development of the mammary gland duct system in mice (Rogler et al., 2009), although miR-374b, miR-362, miR-29, miR-181a, and miR-125b in dairy cows (Li et al., 2012); miR-103, miR-30a-5p, miR-148a, and miR143 in swamp buffalos (Cai et al., 2017); and miR-200 in sheep (Galio et al., 2013) appear to have different expression levels in the different mammary gland development stages. It is therefore suggested that the circular RNAs found in the study may play key roles in mammary gland development. It is interesting to note that some upregulated circular RNAs had higher expression levels at peak lactation than during the nonlactating period in this study, yet in buffalo, the potential target microRNAs such as miR-103, miR-30a-5p, and miR-148a had similar levels of expression during lactation compared with the nonlactating period (Cai et al., 2017). A similar consistent pattern of microRNA expression has been described for miR-125b and miR181a in dairy cows (Li et al., 2012). Overall, there have been no definitive reports describing the relationship between circular RNA expression levels and target microRNA levels, so this will need to be studied in future.

\section{ACKNOWLEDGMENTS}

This work was financially supported by the National Natural Science Foundation of China (32060746, 31860635), the Fuxi Young Talents Fund of Gansu Agricultural University (Gaufx-02Y02), and the fund for Basic Research Creative Groups of Gansu Province (18JR3RA190). The authors have not stated any conflicts of interest.

\section{REFERENCES}

Cai, X., Q. Liu, X. Zhang, Y. Ren, X. Lei, S. Li, Q. Chen, K. Deng, P. Wang, H. Zhang, and D. Shi. 2017. Identification and analysis of the expression of microRNA from lactating and nonlactating mammary glands of the Chinese swamp buffalo. J. Dairy Sci. 100:1971-1986. https://doi.org/10.3168/jds.2016-11461.

Carrington, J. C., and V. Ambros. 2003. Role of microRNAs in plant and animal development. Science 301:336-338. https://doi.org/10.1126/science.1085242.

Chong, B. M., P. Reigan, K. D. Mayle-Combs, D. J. Orlicky, and J. L. McManaman. 2011. Determinants of adipophilin function in milk lipid formation and secretion. Trends Endocrinol. Metab. 22:211-217. https://doi.org/10.1016/j.tem.2011.04.003.

Dai, W. T., Y. X. Zou, R. R. White, J. X. Liu, and H. Y. Liu. 2018. Transcriptomic profiles of the bovine mammary gland during lactation and the dry period. Funct. Integr. Genomics 18:125-140. https://doi.org/10.1007/s10142-017-0580-x.

Finnerty, J. R., W. X. Wang, S. S. Hébert, B. R. Wilfred, G. Mao, and P. T. Nelson. 2010. The miR-15/107 group of microRNA genes: Evolutionary biology, cellular functions, and roles in human diseases. J. Mol. Biol. 402:491-509. https://doi.org/10 .1016/j.jmb.2010.07.051.

Galio, L., S. Droineau, P. Yeboah, H. Boudiaf, S. Bouet, S. Truchet, and E. Devinoy. 2013. MicroRNA in the ovine mammary gland during early pregnancy: Spatial and temporal expression of miR21, miR-205, and miR-200. Physiol. Genomics 45:151-161. https:/ /doi.org/10.1152/physiolgenomics.00091.2012.

Gao, Y., M. Wu, Y. Fan, S. Li, Z. Lai, Y. Huang, X. Lan, C. Lei, H. Chen, and R. Dang. 2018. Identification and characterization of circular RNAs in Qinchuan cattle testis. R. Soc. Open Sci. 5:180413. https://doi.org/10.1098/rsos.180413.

Guo, J. U., V. Agarwal, H. Guo, and D. P. Bartel. 2014. Expanded identification and characterization of mammalian circular RNAs. Genome Biol. 15:409. https://doi.org/10.1186/s13059-014-0409-z.

Hansen, T. B., T. I. Jensen, B. H. Clausen, J. B. Bramsen, B. Finsen, C. K. Damgaard, and J. Kjems. 2013. Natural RNA circles func- 
tion as efficient microRNA sponges. Nature 495:384-388. https:// doi.org/10.1038/nature11993.

Hao, Z., H. Zhou, J. G. H. Hickford, H. Gong, J. Q. Wang, J. Hu, X. Liu, S. Li, M. Zhao, and Y. Luo. 2019. Transcriptome profile analysis of mammary gland tissue from two breeds of lactating sheep. Genes (Basel) 10:781. https://doi.org/10.3390/genes10100781.

Hao, Z., H. Zhou, J. G. H. Hickford, H. Gong, J. Wang, J. Hu, X. Liu, S. Li, M. Zhao, and Y. Luo. 2020. Identification and characterization of circular RNA in lactating mammary gland from two breeds of sheep with different milk production profiles using RNA-Seq. Genomics 112:2186-2193. https://doi.org/10.1016/j.ygeno.2019.12 .014 .

Harris, S., S. Ali, S. Anderson, A. L. Archibald, and A. J. Clark. 1988. Complete nucleotide sequence of the genomic ovine betalactoglobulin gene. Nucleic Acids Res. 16:10379-10380. https:// doi.org/10.1093/nar/16.21.10379.

Hentze, M. W., and T. Preiss. 2013. Circular RNAs: Splicing's enigma variations. EMBO J. 32:923-925. https://doi.org/10.1038/ emboj.2013.53.

Huang, M., Y. Shen, H. Mao, L. Chen, J. Chen, X. Guo, and N. Xu. 2018. Circular RNA expression profiles in the porcine liver of two distinct phenotype pig breeds. Asian-Australas. J. Anim. Sci. 31:812-819. https://doi.org/10.5713/ajas.17.0651.

Jeck, W. R., J. A. Sorrentino, K. Wang, M. K. Slevin, C. E. Burd, J. Liu, W. F. Marzluff, and N. E. Sharpless. 2013. Circular RNAs are abundant, conserved, and associated with ALU repeats. RNA 19:141-157. https://doi.org/10.1261/rna.035667.112.

Ji, Z., G. Wang, Z. Xie, C. Zhang, and J. Wang. 2012. Identification and characterization of microRNA in the dairy goat (Capra hircus) mammary gland by Solexa deep-sequencing technology. Mol. Biol. Rep. 39:9361-9371. https://doi.org/10.1007/s11033-012-1779-5.

Kendziorski, C., R. A. Irizarry, K.-S. Chen, J. D. Haag, and M. N. Gould. 2005. On the utility of pooling biological samples in microarray experiments. Proc. Natl. Acad. Sci. USA 102:4252-4257. https://doi.org/10.1073/pnas.0500607102.

Lasda, E., and R. Parker. 2014. Circular RNAs: Diversity of form and function. RNA 20:1829-1842. https://doi.org/10.1261/rna.047126 .114 .

Legnini, I., G. Di Timoteo, F. Rossi, M. Morlando, F. Briganti, O. Sthandier, A. Fatica, T. Santini, A. Andronache, M. Wade, P. Laneve, N. Rajewsky, and I. Bozzoni. 2017. Circ-ZNF609 is a circular RNA that can be translated and functions in myogenesis. Mol. Cell 66:22-37.e9. https://doi.org/10.1016/j.molcel.2017.02.017.

Li, C., X. Li, Q. Ma, X. Zhang, Y. Cao, Y. Yao, S. You, D. Wang, R. Quan, X. Hou, Z. Liu, Q. Zhan, L. Liu, M. Zhang, S. Yu, W. Ni, and S. Hu. 2017a. Genome-wide analysis of circular RNAs in prenatal and postnatal pituitary glands of sheep. Sci. Rep. 7:16143. https://doi.org/10.1038/s41598-017-16344-y.

Li, C., X. Li, Y. Yao, Q. Ma, W. Ni, X. Zhang, Y. Cao, W. Hazi, D. Wang, R. Quan, X. Hou, Z. Liu, Q. Zhan, L. Liu, M. Zhang, S. Yu, and S. Hu. 2017b. Genome-wide analysis of circular RNAs in prenatal and postnatal muscle of sheep. Oncotarget 8:97165-97177. https://doi.org/10.18632/oncotarget.21835.

Li, Z., C. Huang, C. Bao, L. Chen, M. Lin, X. Wang, G. Zhong, B. Yu, W. Hu, L. Dai, P. Zhu, Z. Chang, Q. Wu, Y. Zhao, Y. Jia, P. $\mathrm{Xu}, \mathrm{H}$. Liu, and G. Shan. 2015. Exon-intron circular RNAs regulate transcription in the nucleus. Nat. Struct. Mol. Biol. 22:256264. https://doi.org/10.1038/nsmb.2959

Li, Z., H. Liu, X. Jin, L. Lo, and J. Liu. 2012. Expression profiles of microRNAs from lactating and non-lactating bovine mammary glands and identification of miRNA related to lactation. BMC Genomics 13:731. https://doi.org/10.1186/1471-2164-13-731.

Livak, K. J., and T. D. Schmittgen. 2001. Analysis of relative gene expression data using real-time quantitative PCR and the $2^{-\Delta \Delta \mathrm{Ct}}$ method. Methods 25:402-408. https://doi.org/10.1006/meth.2001 .1262 .

Memczak, S., M. Jens, A. Elefsinioti, F. Torti, J. Krueger, A. Rybak, L. Maier, S. D. Mackowiak, L. H. Gregersen, M. Munschauer, A. Loewer, U. Ziebold, M. Landthaler, C. Kocks, F. le Noble, and N. Rajewsky. 2013. Circular RNAs are a large class of animal RNAs with regulatory potency. Nature 495:333-338. https://doi.org/10 .1038 /nature11928.

Miranda, K. C., T. Huynh, Y. Tay, Y. S. Ang, W. L. Tam, A. M. Thomson, B. Lim, and I. Rigoutsos. 2006. A pattern-based method for the identification of MicroRNA binding sites and their corresponding heteroduplexes. Cell 126:1203-1217. https://doi.org/10 .1016/j.cell.2006.07.031.

Paten, A. M., E. J. Duncan, S. J. Pain, S. W. Peterson, P. R. Kenyon, H. T. Blair, and P. K. Dearden. 2015. Functional development of the adult ovine mammary gland - insights from gene expression profiling. BMC Genomics 16:748. https://doi.org/10.1186/s12864 -015-1947-9

Paten, A. M., S. J. Pain, S. W. Peterson, H. T. Blair, P. R. Kenyon, P. K. Dearden, and E. J. Duncan. 2014. Identification of reference genes for RT-qPCR in ovine mammary tissue during late pregnancy and lactation and in response to maternal nutritional programming. Physiol. Genomics 46:560-570. https://doi.org/10 .1152 /physiolgenomics.00030.2014.

Rajkumar, A. P., P. Qvist, R. Lazarus, F. Lescai, J. Ju, M. Nyegaard, O. Mors, A. D. Børglum, Q. Li, and J. H. Christensen. 2015. Experimental validation of methods for differential gene expression analysis and sample pooling in RNA-seq. BMC Genomics 16:548. https://doi.org/10.1186/s12864-015-1767-y.

Rogler, C. E., L. Levoci, T. Ader, A. Massimi, T. Tchaikovskaya, R. Norel, and L. E. Rogler. 2009. MicroRNA-23b cluster microRNAs regulate transforming growth factor-beta/bone morphogenetic protein signaling and liver stem cell differentiation by targeting Smads. Hepatology 50:575-584. https://doi.org/10.1002/hep .22982 .

Rybak-Wolf, A., C. Stottmeister, P. Glažar, M. Jens, N. Pino, S. Giusti, M. Hanan, M. Behm, O. Bartok, R. Ashwal-Fluss, M. Herzog, L. Schreyer, P. Papavasileiou, A. Ivanov, M. Öhman, D. Refojo, S. Kadener, and N. Rajewsky. 2015. Circular RNAs in the mammalian brain are highly abundant, conserved, and dynamically expressed. Mol. Cell 58:870-885. https://doi.org/10.1016/j.molcel .2015.03.027.

Sanger, H. L., G. Klotz, D. Riesner, H. J. Gross, and A. K. Kleinschmidt. 1976. Viroids are single-stranded covalently closed circular RNA molecules existing as highly base-paired rod-like structures. Proc. Natl. Acad. Sci. USA 73:3852-3856. https://doi.org/ 10.1073/pnas.73.11.3852.

SEQC/MAQC-III Consortium. 2014. A comprehensive assessment of RNA-Seq accuracy, reproducibility and information content by the Sequencing Quality Control Consortium. Nat. Biotechnol. 32:903914. https://doi.org/10.1038/nbt.2957.

Shi, H., J. Zhu, J. Luo, W. Cao, H. Shi, D. Yao, J. Li, Y. Sun, H. Xu, K. Yu, and J. J. Loor. 2015. Genes regulating lipid and protein metabolism are highly expressed in mammary gland of lactating dairy goats. Funct. Integr. Genomics 15:309-321. https://doi.org/ 10.1007/s10142-014-0420-1.

Smoot, M. E., K. Ono, J. Ruscheinski, P. L. Wang, and T. Ideker. 2011. Cytoscape 2.8: New features for data integration and network visualization. Bioinformatics 27:431-432. https://doi.org/10 .1093/bioinformatics/btq675.

Suárez-Vega, A., B. Gutiérrez-Gil, C. Klopp, G. Tosser-Klopp, and J. J. Arranz. 2016. Comprehensive RNA-Seq profiling to evaluate lactating sheep mammary gland transcriptome. Sci. Data 3:160051. https://doi.org/10.1038/sdata.2016.51.

Sun, J., H. Zhang, B. Hu, Y. Xie, D. Wang, J. Zhang, T. Chen, J. Luo, S. Wang, Q. Jiang, Q. Xi, Z. Chen, and Y. Zhang. 2020. Emerging roles of heat-induced circRNAs related to lactogenesis in lactating sows. Front. Genet. 10:1347. https://doi.org/10.3389/ fgene.2019.01347.

Tang, X., M. G. K. Benesch, and D. N. Brindley. 2015. Lipid phosphate phosphatases and their roles in mammalian physiology and pathology. J. Lipid Res. 56:2048-2060. https://doi.org/10.1194/ jlr.R058362.

Tatarczuch, L., C. Philip, and C. S. Lee. 1997. Involution of the sheep mammary gland. J. Anat. 190:405-416. https://doi.org/10.1046/j 1469-7580.1997.19030405.x. 
Wang, J., H. Zhou, J. G. H. Hickford, Z. Hao, J. Shen, Y. Luo, J. Hu, X. Liu, and S. Li. 2020. Comparison of the transcriptome of the ovine mammary gland in lactating and non-lactating Small-Tailed Han Sheep. Front. Genet. 11:472. https://doi.org/10.3389/fgene .2020.00472.

Wang, L., Z. Feng, X. Wang, X. Wang, and X. Zhang. 2010 DEGseq: An $\mathrm{R}$ package for identifying differentially expressed genes from RNA-seq data. Bioinformatics 26:136-138. https://doi .org/10.1093/bioinformatics/btp612.

Wang, Y., and Z. Wang. 2015. Efficient backsplicing produces translatable circular mRNAs. RNA 21:172-179. https://doi.org/10 $.1261 /$ rna.048272.114.

Wilusz, J. E. 2018. A $360^{\circ}$ view of circular RNAs: From biogenesis to functions. Wiley Interdiscip. Rev. RNA 9:e1478. https://doi.org/ 10.1002 /wrna.1478.

Xu, T., J. Wu, P. Han, Z. Zhao, and X. Song. 2017. Circular RNA expression profiles and features in human tissues: A study using RNA-seq data. BMC Genomics 18(Suppl 6):680. https://doi .org/10.1186/s12864-017-4029-3.
Zhang, C., H. Wu, Y. Wang, Y. Zhao, X. Fang, C. Chen, and H. Chen. 2015. Expression patterns of circular RNAs from primary kinase transcripts in the mammary glands of lactating rats. J. Breast Cancer 18:235-241. https://doi.org/10.4048/jbc.2015.18.3 .235 .

Zhang, C., H. Wu, Y. Wang, S. Zhu, J. Liu, X. T. Fang, and H. Chen. 2016. Circular RNA of cattle casein genes are highly expressed in bovine mammary gland. J. Dairy Sci. 99:4750-4760. https://doi.org/10.3168/jds.2015-10381.

Zhang, Y., X. O. Zhang, T. Chen, J. F. Xiang, Q. F. Yin, Y. H. Xing, S. Zhu, L. Yang, and L. L. Chen. 2013. Circular intronic long noncoding RNAs. Mol. Cell 51:792-806. https://doi.org/10.1016/j .molcel.2013.08.017.

\section{ORCIDS}

Jiqing Wang (ํ) https://orcid.org/0000-0002-9811-0085

Zhiyun Hao 이 https://orcid.org/0000-0002-1296-7038 